\title{
Research on Effect Evaluation for Conceptual Design
}

\author{
Zhigang Xu, Taotao Liu \\ Department of mechanical Engineering, Shandong University, Jinan, 250061, China
}

\begin{abstract}
Keywords: TRIZ, conceptual design, effect library, fuzzy comprehensive evaluation.
\end{abstract}
\begin{abstract}
In conceptual design, for a single functional requirement, various effects or effect chains are correlated with. Designers can only choose effects or effect chains by their own experiences, how ever the inter-disciplinary effects or unfamiliar effects are seldom used. So the effect library are not in full use. Furthermore in the viewpoint of environmental aspect, effects need to rated and classified, so this paper studies similarity differences of effect evaluation. Algorithm on fuzzy comprehensive evaluation is put forward, and calculation of a washing machine is exemplified in this paper.
\end{abstract}

\section{Introduction}

Since twenty-first Century, the product competition renewal speed is getting more and more quickly, product innovation, and or innovative design has become the fundamental guarantee of the survival of enterprises. The characteristics and innovation of products comes mostly from the new physics, chemistry and other effects etc[1-4]. The effect of the knowledge base comes mainly from eight resources: patent, textbooks, monographs, encyclopedias, handbooks, thematic reviews, technical papers and technical reports etc. [5,6]. Effect refers to the use of a certain physical and chemical laws interdisciplinary to solve practical problems in the research works. Certain functions depend on the physical, chemical and geometric effects. Someone puts that certain functions can be achieved through following ways: the single effect model, correlation effect model and controlling effects etc. The above effect model can be combined to effect chains to realize the input and output conversions [7,8].Although effects and/or effect library are getting more and more important nowadays, how ever for a certain function requirement are usually correlated with multiple effects and/or effect chains. How to select a suitable effect, and a selection of evaluation criteria etc are getting more and more important than ever [9]. For this reason, this paper put forward a set of suitable evaluation indexes, evaluation algorithms etc, evaluating cases are exemplified in this paper.

\section{Effect evaluation indexes}

Suitable indexes help to evaluate different effects for conceptual design. According to different design requirements, certain parameters, such as product itself, manufacturing difficulties and environmental factors etc, are taken into considerations. In this paper, four evaluating factors, i.e. the economy (Cost), efficiency (benefit), Feasibility, and environmental friendliness (Environment) are put forward. These factors are talked about in detail in the following sections.

(1) Economy Cost, The economy is the resources consumed refers to obtain the certain quantity and quality effect in the process of using the products and other results of the. For different products, the specific factors to consider different, mainly from the aspects of production cost, user cost, social cost consideration. For example, mechanical products, can consider the ease of manufacture, the manufacturing error sensitivity, energy consumption etc..

(2) Benefit, Efficiency means including economic benefit, social benefit and economic benefits mainly refers to the inflow of products on the market brings to the enterprise economic benefits, well social benefits mainly refers to the eco design products to reduce its environmental and human health and safety aspects of the damage, financing and government so as to achieve the enterprise well known, the expansion of the scope of environmental risk weakening the improvement of the relationship, social support, lower cost competitive advantage. 
(3) Feasibility, Feasibility will vary by product, generally including product design after the completion of the reliability, safety, convenient operation, beautiful appearance and so. Different products have different performance requirements, the need for different indicators to measure.

(4) Environmental friendliness, Environment friendly refers to the effect on the environment in the process of use. Including pollution, resources and energy utilization recovery and handling of the situation after the implementation of the design scheme. For the environmental pollution in this aspect, it includes: air pollution, water pollution, solid waste and noise pollution. The evaluation indexes are summarized in table 1.

Tab.1 Evaluation indexes in product design

\begin{tabular}{|l|l|}
\hline $\begin{array}{l}\text { evaluation } \\
\text { indexes }\end{array}$ & Corresponding factors \\
\hline economy & Production cost、 user cost、social cost \\
\hline benefit & Economy benefit、social benefit \\
\hline feasibility & function、 reliability、 safety、operation、outlook \\
\hline $\begin{array}{l}\text { Environmental } \\
\text { friendliness }\end{array}$ & $\begin{array}{l}\text { air pollution, water pollution, solid waste, noise pollution } \\
\text { resources use rate product recycle rate }\end{array}$ \\
\hline
\end{tabular}

Because the evaluating factors, i.e. product importance, use of the environment, cost etc are of different importance, different weights are assigned to them. Weighting of evaluating index is to characterize the relative importance of different factors. The comprehensive evaluation results are obtained by evaluating the product of weights of each index and the index values. Matrix A is used in this paper, which is consisted of weights of evaluation index in the product design process, i.e. $\mathrm{A}=[\mathrm{a} 1 \mathrm{~A} 2 \mathrm{~A} 3 \mathrm{a} 4]$, where A1, A2, A3, A4 respectively, the effect of economic, benefit, feasibility, environmental friendliness in the whole scheme of the weight, and $\mathrm{a} 1+\mathrm{a} 2+\mathrm{a} 3+\mathrm{a} 4=1$. If there are $\mathrm{m}$ experts, who are to define the weight of evaluation index matrix A, this matrix will be recorded as $\mathrm{Ak}=[\mathrm{ak} 1 \mathrm{ak} 2 \mathrm{ak} 3 \mathrm{ak} 4](\mathrm{k}=1,2,3, \ldots \ldots, \mathrm{m})$.

\section{Determination of the comprehensive weight matrix}

Weight distribution is of different usages, but, for the similar products, weight distributions are identical in numbers. Effects are inter-disciplinary, coming from physical, chemical, and many subjects such as mathematics, biological synthesis application etc, so judgment distortion or miscarriage of justice lead to wrong judgment. For this reason, crowd experts are usually gathered to determine weight distributions, and fuzzy comprehensive weight evaluation algorithms are utilized to remove judgment bias due to subjective individual evaluation.

\section{Similarities}

Cosine of the angle between two vectors reflect the similarity of two vectors, i.e. the expert judgment, the smaller the angle, the greater the cosine vector similarity degree, and higher degree of the same experts' opinion, and vice visa. $\xi \mathrm{i}=(\mathrm{ai1}, \mathrm{ai} 2, \mathrm{ai3}, \mathrm{ai} 4) \quad \xi \mathrm{j}=(\mathrm{aj} 1, \mathrm{aj} 2, \mathrm{aj} 3, \mathrm{aj} 4)$, The angle of two vectors is defined by $\theta$, and $\operatorname{cosine}$ of $\theta$ is defined in the following equation.

$$
\cos \theta=\frac{<\xi_{i}, \xi_{j}>}{\left\|\xi_{i}\right\| \xi_{j} \|}=\frac{\sum_{t=1}^{4} a_{i t} a_{j t}}{\sqrt{\sum_{t=1}^{4}\left(a_{i t}\right)^{2}} \sqrt{\sum_{t=1}^{4}\left(a_{j t}\right)^{2}}}
$$

If $\eta \mathrm{ij}=\operatorname{COS} \theta$ is defined by the geometric similarity of the two vectors.So $0 \leq \eta \mathrm{ij} \leq 1$, only when $\xi \mathrm{i}=\xi \mathrm{j}, \eta \mathrm{ij}=1$. 
For a evaluating weighting index matrix from ms'experts, there are ms vectors form the weighting matrix,i.e. $\xi 1 \xi 2 \ldots \xi \mathrm{m}$ etc. Let

$$
\eta^{(i)}=\sum_{j=1}^{m} \eta_{i j}-1
$$

This equation denotes the similarities among vector $\xi \mathrm{i}$ and the other vectors, the larger $\eta(\mathrm{i})$ is, the similar the k's expert's opinion with the other experts are, and the credibility is high. Normalization of $\eta(i)$ 即is utilized to describe the similarity of the i's expert with the others, which is defined as $u(i)$ :

$$
u^{(i)}=\frac{\eta^{(i)}}{\sum_{j=1}^{m} \eta^{(j)}}, \mathrm{i}=1,2, \ldots, \mathrm{m}
$$

\section{Differences}

There are a number of vectors, i.e, $\xi 1 \xi 2 \ldots \xi \mathrm{m}$, which are abstracted from the weighting matrix. Where, $\xi_{\mathrm{k}}=(\mathrm{ak} 1, \mathrm{ak} 2, \mathrm{ak} 3, \mathrm{ak} 4)$ denotes four evaluation indexes from a certain expert,if ei is the i's average weights, so:

$$
\begin{gathered}
e_{i}=\frac{1}{m} \sum_{k=1}^{m} a_{k i}, \quad \mathrm{i}=1,2,3,4 \\
\text { Let } \sigma_{k i}=\left(a_{k i}-e_{i}\right)^{2}
\end{gathered}
$$

So $\sigma_{k i}$ is the mean variance of the i's evaluating factor from the k's expert, Furthermore, let

$$
\sigma_{k}=\sum_{i=1}^{4} \sigma_{k i}, \mathrm{k}=1,2, \ldots, \mathrm{m} ;
$$

Where $\sigma \mathrm{k}$, the sum of the mean variance of each evaluating factor from the k's expert.

$$
\text { Let } \quad \sigma^{(k)}=\sigma_{k} / \sigma, \mathrm{k}=1,2, \ldots, \mathrm{m} ; \text { where } \sigma=\sum_{k=1}^{m} \sigma_{k}
$$

This is the ratio of the sum of the mean variance of the k's expert with that of the other experts, $\sigma(\mathrm{k})$ denotes the difference of the k's expert, the larger the value, the lower the credibility, and vice visa.

\section{Credibility and the final weight distribution}

Credibility is described by two parameters, i.e., similarity and difference. So credibility $\omega(\mathrm{k})$ is defined as the following equation:

$$
\omega^{(k)}=\frac{u^{(k)}-u^{(k)} \delta^{(k)}}{1-\sum_{k=1}^{m} u^{(k)} \delta^{(k)}}=\frac{u^{(k)}\left(1-\delta^{(k)}\right)}{1-\sum_{k=1}^{m} u^{(k)} \delta^{(k)}}
$$

And the final matrix is defined in equation (9).

$$
A_{0}=\left[\begin{array}{llllll}
\omega^{(1)} & \omega^{(2)} & \ldots & \omega^{(k)} & \ldots & \omega^{(m)}
\end{array}\left[\begin{array}{cccc}
a_{11} & a_{12} & a_{13} & a_{14} \\
a_{21} & a_{21} & a_{23} & a_{24} \\
\ldots & \ldots & \ldots & \ldots \\
a_{k 1} & a_{k 2} & a_{k 3} & a_{k 4} \\
\ldots & \ldots & \ldots & \ldots \\
a_{m 1} & a_{m 2} & a_{m 3} & a_{m 4}
\end{array}\right]\right.
$$


Where $\mathrm{k}=1,2, \ldots, \mathrm{m}$.

\section{Examples}

Washing machine is now taken as an design example, for a traditional washing machine, detergent is widely used in large quantities, which leads to heavy water pollution. So the environmental factor is set in a larger number. Evaluating factor from different experts are summarized in table1.

Tab.2 Weight distribution of different evaluating factors

\begin{tabular}{|l|l|l|l|l|}
\hline Expert team & economy & benefit & feasibility & Environment friendliness \\
\hline Expert 1 & 0.10 & 0.30 & 0.20 & 0.40 \\
\hline Expert 2 & 0.15 & 0.30 & 0.15 & 0.40 \\
\hline Expert 3 & 0.15 & 0.25 & 0.20 & 0.40 \\
\hline Expert 4 & 0.15 & 0.30 & 0.20 & 0.35 \\
\hline
\end{tabular}

So weighting vectors are as the followings:

$$
\xi 1=\left(\begin{array}{llll}
0.10 & 0.30 & 0.20 & 0.40
\end{array}\right) \quad \xi 2=\left(\begin{array}{llll}
0.15 & 0.30 & 0.15 & 0.40
\end{array}\right) \xi 3=\left(\begin{array}{lllll}
0.15 & 0.25 & 0.20 & 0.40
\end{array}\right) \quad \xi 4=\left(\begin{array}{lll}
0.15 & 0.30
\end{array}\right.
$$
$0.200 .35)$

By equation (1)(2), we can get that: $\quad \eta(1)=2.9756 \quad \eta(2)=2.9749 \quad \eta(3)=2.9745 \quad \eta(4)=2.9752$

By equation (3), similarities are obtained. $\quad u(1)=0.25005 \quad u(2)=0.24999 \quad u(3)=0.24995$ $u(4)=0.25001$

By equation (4)(5)(6), we can know that: $\sigma(1)=0.001875 \quad \sigma(2)=0.001875 \quad \sigma(3)=0.001875$ $\sigma(4)=0.001875$

By equation (7), differences are obtained. $\quad \delta(1)=0.25 \quad \delta(2)=0.25 \quad \delta(3)=0.25 \quad \delta(4)=0.25$

By equation (8), credibility factors are obtained. $\omega(1)=0.25005 \omega(2)=0.24999 \omega(3)=0.24995$ $\omega(4)=0.25001$

And the final weigh distribution matrix A0 is obtained.

$$
A_{0}=\left[\begin{array}{llll}
0.25005 & 0.24999 & 0.24995 & 0.25001
\end{array}\right]\left[\begin{array}{llll}
0.10 & 0.30 & 0.20 & 0.40 \\
0.15 & 0.30 & 0.15 & 0.40 \\
0.15 & 0.25 & 0.20 & 0.40 \\
0.15 & 0.30 & 0.20 & 0.35
\end{array}\right]
$$

Where $\mathrm{A} 0=\left[\begin{array}{llll}0.1375 & 0.2875 & 0.1875 & 0.3875\end{array}\right]$

Weights of economic feasibility, benefits, environmental friendliness, is $0.1375,0.2875,0.1875$, 0.3875 respectively. In the conceptual design stage, fuzzy evaluation is introduced. And the evaluating index is divided into four degrees, i.e. good, fair good, general, poor, very poor in five degrees of scoring.

An expert group is introduced in the evaluation of ultrasonic washing machine, where four factors, i.e. feasibility, economy, benefit, environment friendliness are taking into consideration. Scores are recorded in table 2.

Tab. 2 results of evaluating indexes

\begin{tabular}{|l|l|l|l|l|l|}
\hline evaluating indexes & good & Fair good & fair & Fair poor & poor \\
\hline economy & 0.20 & 0.40 & 0.40 & 0 & 0 \\
\hline benefit & 0.40 & 0.30 & 0.20 & 0.10 & 0 \\
\hline feasibility & 0.30 & 0.40 & 0.30 & 0 & 0 \\
\hline Environ-friendliness & 0.50 & 0.40 & 0.10 & 0 & 0 \\
\hline
\end{tabular}

The evaluating results is put in matrix $\mathrm{J}$, as the followings. 


$$
J=\left[\begin{array}{ccccc}
0.20 & 0.40 & 0.40 & 0 & 0 \\
0.40 & 0.30 & 0.20 & 0.10 & 0 \\
0.30 & 0.40 & 0.30 & 0 & 0 \\
0.50 & 0.40 & 0.10 & 0 & 0
\end{array}\right]
$$

The final results is denoted by $\mathrm{T}$ in the followings.

$$
\begin{aligned}
T=A_{0}^{*} J & =\left[\begin{array}{llll}
0.1375 & 0.2875 & 0.1875 & 0.3875
\end{array}\right]\left[\begin{array}{cccccc}
0.20 & 0.40 & 0.40 & 0 & 0 \\
0.40 & 0.30 & 0.20 & 0.10 & 0 \\
0.30 & 0.40 & 0.30 & 0 & 0 \\
0.50 & 0.40 & 0.10 & 0 & 0
\end{array}\right] \\
& =\left[\begin{array}{lllll}
0.39250 & 0.37125 & 0.20750 & 0.02875 & 0
\end{array}\right]
\end{aligned}
$$

If $100,75,50,25,0$ are used to denote five degrees of "good,fair good,fair,fair poor, poor", so, $F=\left[\begin{array}{lll}0.3925 & 0.371250 .20750 .02875\end{array}\right]\left[\begin{array}{c}100 \\ 75 \\ 50 \\ 25 \\ 0\end{array}\right]=78.175$

The final result is 78.1875 for this evaluating example, and this effect can be considered in innovative design of washing machines.

\section{Conclusions}

Aiming at the utilization of effects in conceptual design, the fuzzy comprehensive evaluation algorithms are put forward. Similarities and differences between different experts are talked about in the evaluation processes, factors as well as algorithms are introduced and talked about in detail.Evaluating examples is used to illustrate the effectiveness of this method.

\section{Acknowledgments}

The above-mentioned research work is supported by the Chinese NSFC, 61272017, P.R. China, and the Key Laboratory of High-efficiency and Clean Mechanical Manufacture at Shandong University, Ministry of Education.

\section{References}

[1] H Dou, V Leveill, S Manullang, et al, Patent analysis for competitive technical intelligence and innovative thinking. Data science journal, 2005, 4 (3) : 209 237.

[2] Magerman T, Van Looy B, Song XY. Exploring the feasibility and accuracy of Latent Semantic Analysis based text mining techniques to detect similarity between patent documents and scientific publications. Scien to metrics, 2010, 82( 2 ), 289 306.

[3] Q iu Qing ying, Wang Zhao Xia, Feng Peien, et al, Working structure knowledge acquisition from mechanical product patent based on natural language understanding, 2009WRI World Congress on Computer Science and Information Engineering, Los Angeles, USA, 2009: 195 199.

[4] Zhenxin Cao, Haoxing Zhao. Research of knowledge acquisition and modeling method based on patent map, 2008 IEEE International Symposium on Knowledge Acquisition and Modeling Workshop, Wuhan, China, 2008: 1090 1094.

[5] Sungjoo Lee, Seonghoon Lee, Hyeonju Seol, et al, Using patent information for designing new product and technology: keyword based technology road mapping. R\& D Management, 2008,38( 2 ) : 169 188. 
[6] TAN Runhua. Progress of some problems in product design for innovation. Chinese Journal of Mechanical Engineering, 2003, 39 (9) : 11- 16.

[7] SHEN Minde, FEN G Peien, SONGYe. Construction of mechanics-effects-based knowledge bank used in conceptual design for mechanical transmission system. Journal of Engineering Design, 1999, (2) : 11- 16.

[8] Yan Li, Jian Wang, Xianglong Li, et al, Design creativity in product innovation. The International Journal of Advanced Manufacturing Technology, 2007, 33( 3- 4 ), 213 222.

[9] W. Y. Zhang, S. B. Tor, G. A. Britton and Y.-M. Deng. A Knowledge-Based Expert System for Functional Design of Engineering Systems. Engineering with Computers (2001) 17: 339-353. 